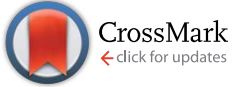

Cite this: RSC Adv., 2015, 5, 78677

Received 10th June 2015

Accepted 9th September 2015

DOI: $10.1039 / \mathrm{c} 5 \mathrm{ra11032g}$

www.rsc.org/advances

\section{Understanding the formation of PEDOT:PSS films by ink-jet printing for organic solar cell applications}

\begin{abstract}
Arjun Singh, ${ }^{\text {ab }}$ Monica Katiyar ${ }^{\mathrm{ab}}$ and Ashish Garg ${ }^{\star a b}$
One of the most exciting aspects of organic electronic devices, in particular organic solar cells, is their potential for low cost and large scale manufacturing using printing technologies such as inkjet printing. In this manuscript, we report our work on developing an understanding of the ink-jet printing process to print smooth and transparent PEDOT:PSS (poly(3,4-ethylenedioxythiophene):poly(styrene sulfonate)) films for organic solar cell devices. The parameters that were found to strongly affect the formation of a continuous PEDOT:PSS film were substrate surface treatment, drop spacing, substrate temperature during printing as well as annealing. We investigated the effect of these parameters through detailed morphological characterization using optical and atomic force microscopy and the results suggested that one can achieve a transmittance of $\sim 90 \%$ for a ca. $110 \mathrm{~nm}$ thick film with a uniform structure and morphology, fabricated using a drop spacing of $30 \mu \mathrm{m}$ and an annealing temperature of $120{ }^{\circ} \mathrm{C}$. Overall, PEDOT:PSS film characteristics are strongly influenced by the substrate temperature during printing and annealing temperature as well as surface modification, determined by a mix of surface wetting characteristics, rate of evaporation of the solvent and coalescence of the printed lines. The organic solar devices fabricated using these inkjet printed PEDOT:PSS films show comparable performance yielding a power conversion efficiency of ca. $2.64 \%$ in comparison to ca. $3.1 \%$, showed by the devices fabricated on standard spin coated PEDOT:PSS films.
\end{abstract}

\section{Introduction}

One of the reasons organic electronics has caught enormous attention from the research community as well as industry is the manufacturability of organic devices using various low cost mass production techniques such as printing which could potentially result in high throughput low cost device fabrication. There are various printing processes which are being considered for printing of organic materials and other device components such as ink-jet printing, dip_coating ${ }^{1}$ gravure printing, doctor blading and screen printing. ${ }^{2}$ Among these, ink-jet printing has a few advantages over other printing methods such as non-contact printing, mask-less and combinatorial processing with very little wastage, easy programmability and a reasonable speed of operation.

Ink-jet printing consists of various steps leading to the formation of a continuous film: first the formation of an ink followed by its jetting and spreading on the substrate surface followed by its subsequent solidification. One of the key parameters affecting ink-jet printing is the formation of drops from the jets of liquid. ${ }^{3,4}$ The drop formation and subsequent

${ }^{a}$ Department of Materials Science and Engineering, Indian Institute of Technology Kanpur, Kanpur-208016, India. E-mail: ashishg@iitk.ac.in

${ }^{b}$ Samtel Centre for Display Technologies, Indian Institute of Technology Kanpur, Kanpur-208016, India print quality of the films strongly depends on various properties of the ink as well as substrate characteristics. The parameters relevant to the ink are its viscosity and surface tension, while spreading and wetting of jetted drops depend on the jetting voltage, characteristics of the substrate surface ${ }^{5}$ and substrate temperature. Further film drying behavior has a critical effect on final surface uniformity. Even though the surface of initial liquid film may be flat, the surface of the dried film may not be uniformly flat. These parameters are often system specific and hence there is a need to understand the effect of these parameters on the film formation in the context of materials being printed.

Printing methods are also being considered as potential manufacturing processes for organic solar cells (OSC), particularly from the perspective of roll-to-roll and higher throughput low cost processing. PEDOT:PSS (poly(3,4ethylenedioxythiophene):poly(styrene sulfonate)) (PEDOT:PSS) is an important polymeric material because of high optical transparence in the visible region, flexibility, high work function and easy to solution process. However poor conductivity of PEDOT:PSS limits its use in organic electronic devices. The additives (e.g. DMSO, ${ }^{6}$ glycerol $\left.{ }^{7}\right)$ or surface treatment (e.g. meth$\mathrm{anol}^{8}$ ) are used for improving the device performance by enhancing its conductivity and controlled morphology as well. A few reports also show the variation in the thickness also influences the device behaviour. ${ }^{9-11}$ Besides applications in organic 
solar cells, modified PEDOT:PSS can also be used for thermoelectric devices, ${ }^{\mathbf{1 2 - 1 4}}$ lithium ion batteries ${ }^{15}$ and hybrid solar cells. ${ }^{16}$ While printing techniques are suitable for large area application of OPVs, there are a few challenges associated with area scaling due to high sheet resistance of transparent conducting electrodes e.g. indium tin oxide (ITO) ${ }^{17,18}$ as well as increased number for leakage paths in large area devices because of randomly distributed defects in thin films ${ }^{19}$ and thickness variations. ${ }^{2021}$ Although attempts have been made on alternate electrode structure such as insulator-metal-insulator structure for monolithic submodules to reduce the series resistance of electrodes, the efficiencies are still low. ${ }^{22}$

A typical device structure that is used is a bulk heterojunction configuration where a blend of light absorbing polymer (e.g. P3HT or poly-3-hexylthiophene) and an acceptor, typically $\mathrm{PC}_{60} \mathrm{BM}$, a fullerene, are sandwiched between the two electrodes. Between the electrodes and the blend layer (also called as active layer), often there are thin layers which provide selectivity to the carrier transport towards cathode (e.g. Al) and the anode (indium tin oxide or ITO). In organic solar cell devices with normal architecture, a typical configuration is glass/ITO/ PEDOT:PSS/P3HT:PC ${ }_{60} \mathrm{BM} / \mathrm{Ca} / \mathrm{Al}$. Here, a thin poly(3,4ethylenedioxythiophene):poly(styrenesulfonate) (PEDOT:PSS) layer is used as a hole transport layer for selective flow of holes to the anode. PEDOT:PSS film not only works as a hole transport layer, it also affects the planarization of the underlying ITO electrode as well as interface quality between this layer and the active layer. ${ }^{7}$ Hence, for fabricating a printable device, it is important to understand the printing aspects of the PEDOT:PSS layer and literature is surprisingly devoid of details on understanding the effect of process parameters on printing characteristics of organic materials, PEDOT:PSS in the present context. While a few previous studies report the effect of annealing temperature $^{11}$ and solvent addition to the ink ${ }^{6}$ on the quality of inkjet printed PEDOT:PSS films on glass substrates, a detailed understanding of the formation of PEDOT:PSS films on ITO coated glass substrates is essential for better process control and development.

In this report, we present a detailed study to understand the formation of PEDOT:PSS layer including formation of the ink for efficient jetting and stable drop formation by varying the concentration of its ingredients in the original ink. The dynamic contact angle with time was measured for studying the ink spreading over ITO coated glass substrates with temperature. We have investigated the effect of process parameters such as drop spacing, substrate temperature, substrate surface modification and annealing temperature on the film formation, critical to the formation of a good quality OSC devices. Finally, we demonstrate working P3HT: $\mathrm{PC}_{60} \mathrm{BM}$ organic solar cell devices using printed PEDOT:PSS films with efficiencies reasonably comparable to those obtained on spin coated PEDOT:PSS films.

\section{Experimental}

PEDOT:PSS ink was prepared by diluting commercially available PEDOT:PSS (Clevios P CH8000) with deionized (DI) water in different ratios followed by filtration of the solution using $0.22 \mu \mathrm{m}$ PVDF filter to prevent any nozzle clogging. The ink viscosity was measured using the Ostwald U-shape glass viscometer while surface tension of the substrates and contact angle were measured using OCA135 Data Physics goniometer employing sessile drop method. Prior to the printing of PEDOT:PSS ink, ITO coated glass substrates were cleaned in the soap solution followed by ultrasonication first in DI water and then in the RCA solution (DI $+\mathrm{NH}_{3}+\mathrm{H}_{2} \mathrm{O}_{2}$ mixed in a ratio of $5: 1: 1)$ for 20 minutes followed by rinsing with DI water and drying in flowing $\mathrm{N}_{2}$ gas. The surface treatment of ITO coated glass sample was done by carrying out oxygen plasma treatment (Plasma Preen II-973, Plasmatic Systems Inc) with 5 psi gas pressure and at full power level. The ozone treatment was done with UVO-cleaner (model no. 42-220, Jelight) for 15 minutes.

The printing of PEDOT:PSS films of area upto $10 \times 10 \mathrm{~mm}^{2}$ was carried out using Dimatix 2831 printer having nozzle diameter $21 \mu \mathrm{m}$ and using the waveform provided by Dimatix. The printed area of film was $10 \times 10 \mathrm{~mm}^{2}$ and $1.5 \times 1.5 \mathrm{~mm}^{2}$. A reference PEDOT:PSS sample was prepared by spin coating PEDOT:PSS ink at $2000 \mathrm{rpm}$ on ITO coated glass substrates followed by annealing at $120{ }^{\circ} \mathrm{C}$ for 10 minutes. Surface topography and thickness measurement on the films were carried out using Zeiss optical measurement and KLA Tensor surface profilometer. Roughness was measured using Ambios and Asylum Research atomic force microscopy (AFM) while transmittance spectra were measured using Perkin-Elmer Lambda 750 spectrophotometer.

For solar cell device fabrication on PEDOT:PSS (printed as well as spin coated) coated substrates, first the blend solution of $\mathrm{P} 3 \mathrm{HT}$ and $\mathrm{PC}_{60} \mathrm{BM}$ was prepared in chlorobenzene with concentration $15 \mathrm{mg} \mathrm{ml} \mathrm{m}^{-1}$ each in a $\mathrm{N}_{2}$ filled glove box. Subsequently, the solution was spin coated on PEDOT:PSS coated substrates at 2000 rpm yielding a film thickness of $c a .100 \mathrm{~nm}$. Subsequently, LiF $(0.8 \mathrm{~nm})$ and aluminum $(100 \mathrm{~nm})$ were evaporated using a thermal evaporator attached with the glove box. Current-voltage measurements of the device were carried out using Keithley 2400 source meter at $1.5 \mathrm{G}$ solar spectrum and a light intensity of 100 $\mathrm{mW} \mathrm{cm}^{-2}$ using a Newport solar simulator.

\section{Results and discussion}

\subsection{Effect of viscosity on drop formation}

Prior to the printing of PEDOT:PSS films, it was essential to form an ink which does not give rise to any satellite drop formation which follows the leading drop from the nozzle upon jetting. These satellite drops are easily misdirected and can reduce the quality of the pattern to be printed. The dynamics of ink droplet formation depends on various physical properties i.e. density, viscosity, surface tension of the ink. Most important of these is the viscosity which is manifested in the inverse of dimensionless Fromm number, $Z$ and is given as

$$
Z=\frac{(a \rho \gamma)^{1 / 2}}{\eta}
$$

where $a$ is the radius of the printing orifice, $\eta$ is the viscosity, $\gamma$ is the surface tension and $\rho$ is the density of the ink. From the 
perspective of drop formation without a satellite, whilst a value of $Z>2$ was theoretically predicted by Fromm et al., ${ }^{23}$ recent works $^{2}$ suggest that a range of $4 \leq Z \leq 14$ is more appropriate.

We controlled the viscosity of the ink by changing the volume ratio of PEDOT:PSS to DI water using three combinations: $1: 2,1: 1$ and $2: 1$ leading to a $Z$ value of 6.72 (viscosity $3.95 \mathrm{cP}$ ), 5.37 (viscosity $\sim 4.9 \mathrm{cP}$ ) and 4.63 (viscosity $\sim 5.65 \mathrm{cP}$ ) which are in the range predicted by Fromm et al. As Fig. 1 shows, with increase in the ink viscosity, the time to form a spherical drop with satellite formation is reduced. At PEDOT:PSS to DI water ratio of $2: 1(\eta=5.65 \mathrm{cP})$, a spherical drop is formed after an elapsed time of $45 \mu \mathrm{s}$ while spherical drops are formed after $65 \mu$ s for a ratio of $1: 1(\eta=4.95 \mathrm{cP})$. We do not see formation of a spherical drop at the ratio of $1: 2(\eta=$ $3.95 \mathrm{cP}$ ) within the camera resolution of the printer. Based on these results, we selected an ink viscosity of $5.65 \mathrm{cP}(2: 1)$ for printing experiments.

3.2 Effect of drop spacing and the substrate temperature vis$\grave{a}$-vis surface treatment on the formation of PEDOT:PSS film

First, we printed PEDOT:PSS films on the ITO coated glass substrates which were only cleaned with no further surface treatment. The films were printed at the drop spacing of $30 \mu \mathrm{m}$, $33 \mu \mathrm{m}$ and $35 \mu \mathrm{m}$ drop spacing and at substrate temperatures of $30{ }^{\circ} \mathrm{C}, 35{ }^{\circ} \mathrm{C}$ and $40{ }^{\circ} \mathrm{C}$. Fig. 2 shows the optical micrographs of these films comparing their morphologies. These micrographs show that neither the variation in the drop spacing nor in substrates temperature yields a continuous film. The lines tend to appear sharper as the temperature of the substrate is increased at any drop spacing which is due to increased evaporation rate of the solvent. However, in none of the films, lines showed a tendency to merge and form a continuous film suggesting that the spreading of the ink is not uniform over the substrate surface and could possibly be related to the poor wetting i.e. higher contact angle of the ink on the untreated substrate.

Based on the above results, we carried out surface treatment of the ITO coated glass substrates by exposing them to oxygen plasma treatment for various times and investigated the effect on the film morphology with respect to drop spacing and the substrate temperature. Surface treatments such as oxygen plasma or UV-ozone treatment of ITO surface are believed to improve the ITO surface wettability to aqueous solutions ${ }^{24}$ without leading to any etching effects. However, their effect with respect to printing of organic films is not clearly reported. Fig. 3
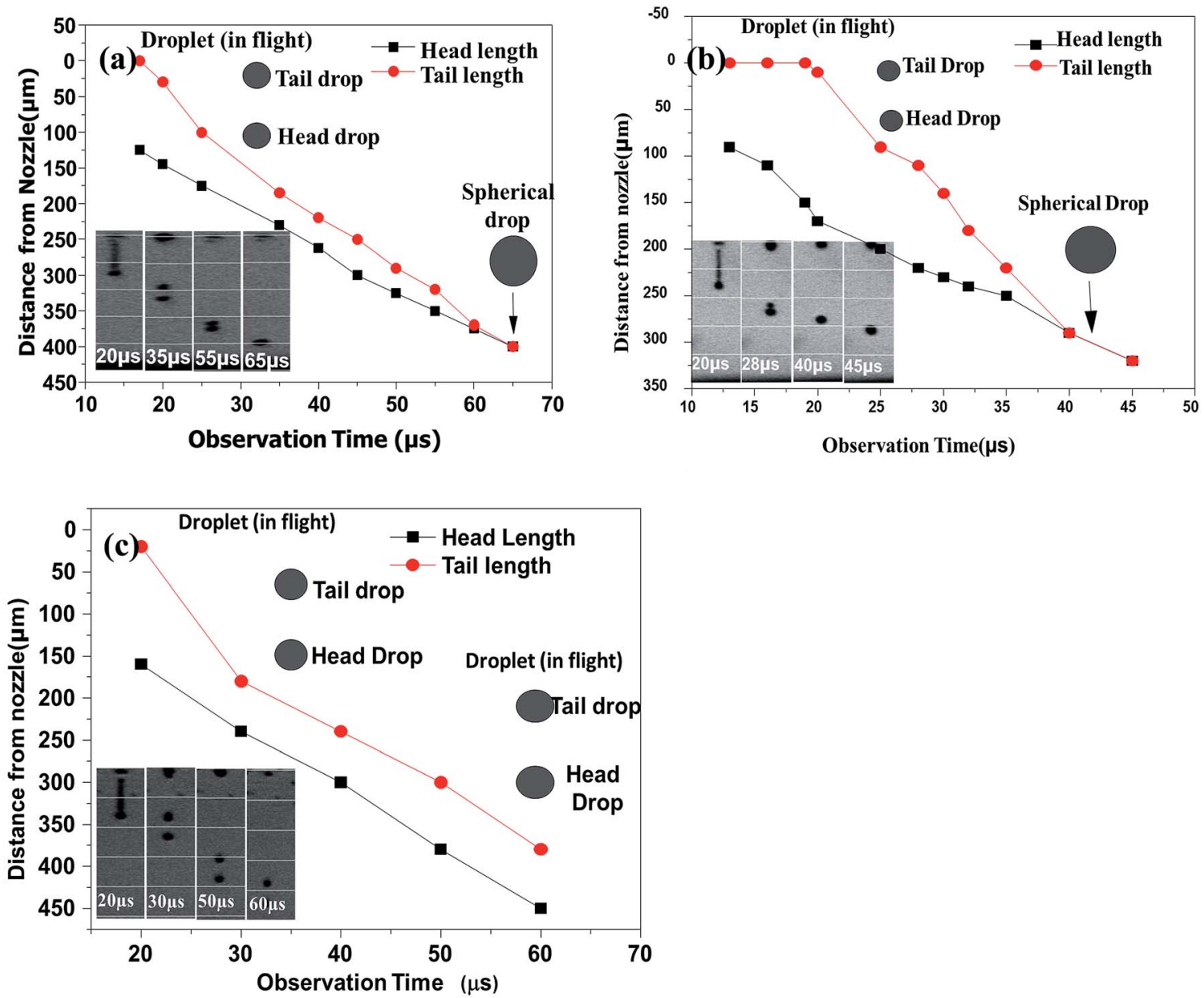

Fig. 1 Effect of PEDOT:PSS : DI water volume ratio on the ink jetted drop formation (a) PEDOT:PSS : DI (1 : 1), (b) PEDOT:PSS : DI (2 : 1), (c) PEDOT:PSS : DI (1:2) (insets show transient behavior of the drops). 


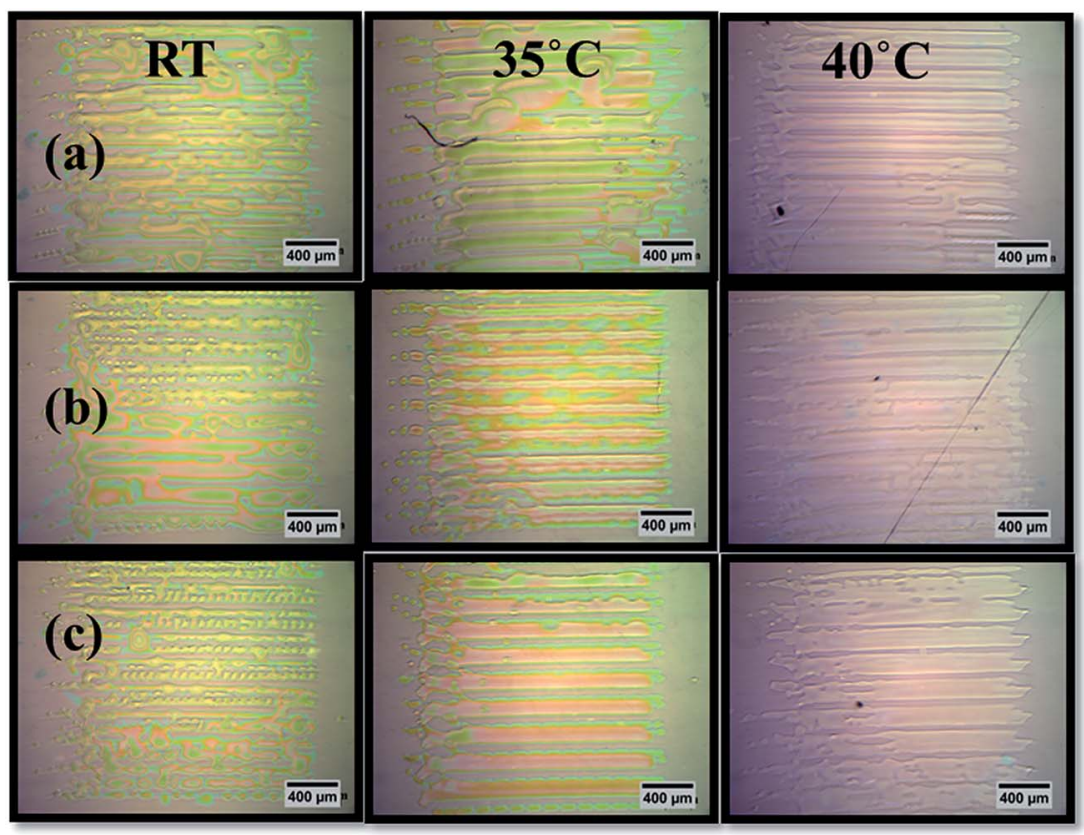

Fig. 2 Optical microscope images of PEDOT:PSS films printed on ITO coated glass substrates with no prior surface treatment at different drop spacing: (a) $30 \mu \mathrm{m}$ (b) $33 \mu \mathrm{m}$ (c) $35 \mu \mathrm{m}$ and different substrate temperature $\left(T_{\mathrm{s}}\right)$.

shows the optical micrographs of inkjet printed PEDOT:PSS films on ITO coated glass substrates which were oxygen plasma treated for $1 \mathrm{~min}$ and $3 \mathrm{~min}$. Upon comparison with Fig. 2, it is evident that with the oxygen plasma treatment, the coverage of the ink and hence film uniformity improves dramatically. The lines only appear rather unclearly for the drop spacing of $35 \mu \mathrm{m}$ while they are not at all seen for the drop spacing of 33 and $30 \mu \mathrm{m}$. Moreover, the morphology of films with 3 min plasma treatment is much better than for
1 min treatment suggesting that oxygen plasma treatment leads to a decrease in the contact angle of the ink on the substrate and hence better wettability leading to an improved coverage of the substrate. Fig. 3 shows the complete printed pad whose area is small, i.e. $1.5 \times 1.5 \mathrm{~mm}^{2}$ whose objective was to optimize the oxygen plasma time. The edges are not very sharp which we believe is due to splashing of drops during printing. Hence for subsequent experiments, we chose the oxygen plasma treatment time of 3 minutes.

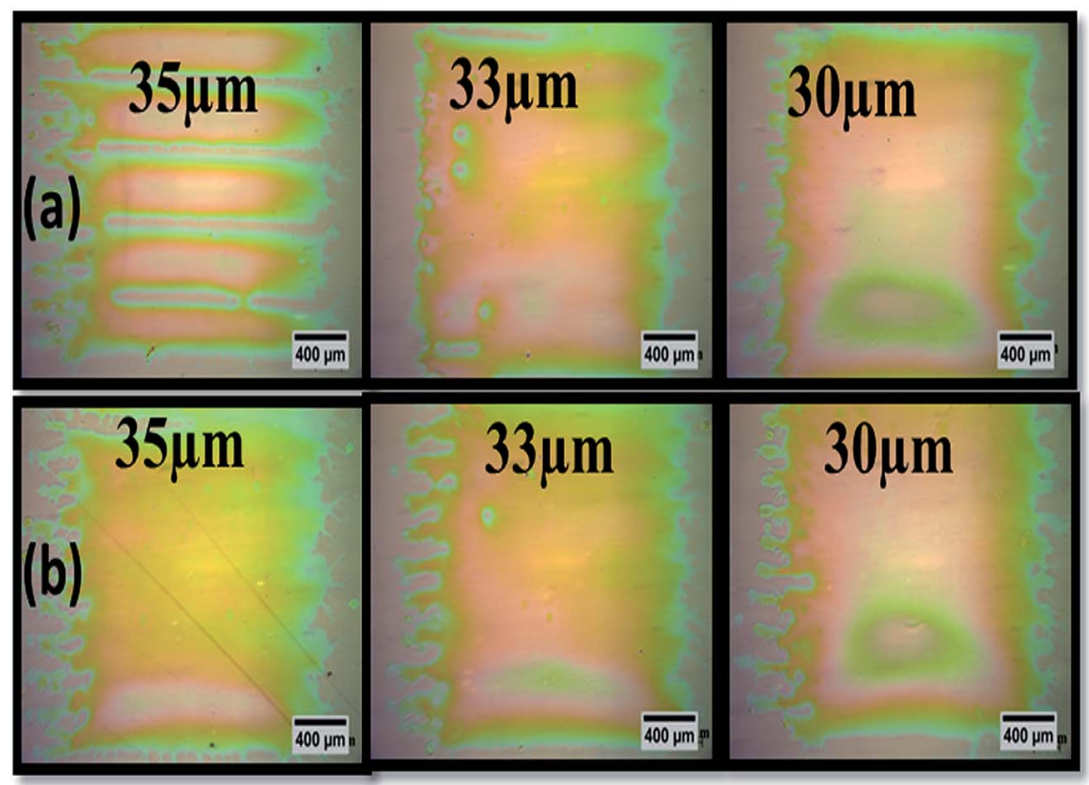

Fig. 3 Optical microscope images of PEDOT:PSS films printed with different drop spacing and oxygen plasma exposure for (a) 1 min and (b) $3 \mathrm{~min}$. 
We also checked the effect of substrate temperature on the printing of PEDOT:PSS films for both oxygen plasma treated substrates as well ozone treated ITO/glass substrates. In both the cases, as shown in Fig. 4, increasing the substrate temperature leads to an increased non-uniformity of the films as at higher temperatures. This indicates that the printed lines do not merge due to faster evaporation of the solvent. This experiment suggested that printing at room temperature $(\mathrm{RT}=22 \pm$ $2{ }^{\circ} \mathrm{C}$ ) yields superior quality films with good uniformity irrespective of the type of surface treatment used. The morphology of these films appears superior to those shown in Fig. 3 because in these experiments, the printed area was large, $12 \times 12 \mathrm{~mm}^{2}$, which allows one to achieve a large enough uniform area avoiding the edge effects.

Finally, to understand the effect of substrates temperature, we carried out dynamic contact angle measurements with time on the substrates treated with oxygen plasma. The results, as shown in Fig. 5, show that at RT, the contact angle decreases with time and becomes stable after $\sim 10 \mathrm{~s}$ whilst at higher substrates temperatures, the contact angle reaches a stable value at lower times, $\sim 4-5$ seconds. This suggests that the spreading of the ink is hindered by substrate temperature and increase in temperature leads to faster evaporation of the ink solvent. This spreading is critical to the film formation and drop

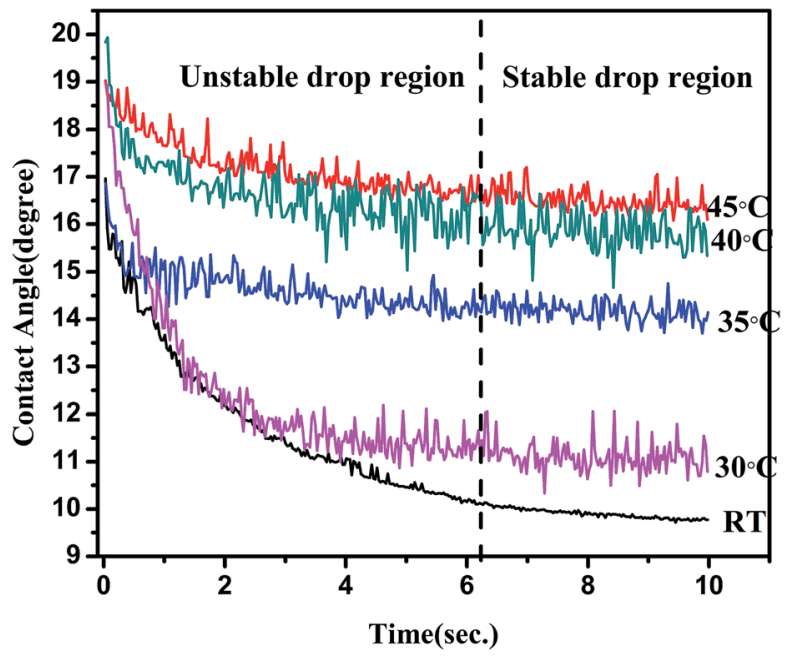

Fig. 5 Dynamic contact angle measurements of PEDOT:PSS ink on oxygen plasma treated ITO coated glass substrates at different substrates temperatures.

spacing. These measurements also show that with increase in the substrates temperature, the contact angle increases resulting in poor spreading of drops as well as a reduction in the drop
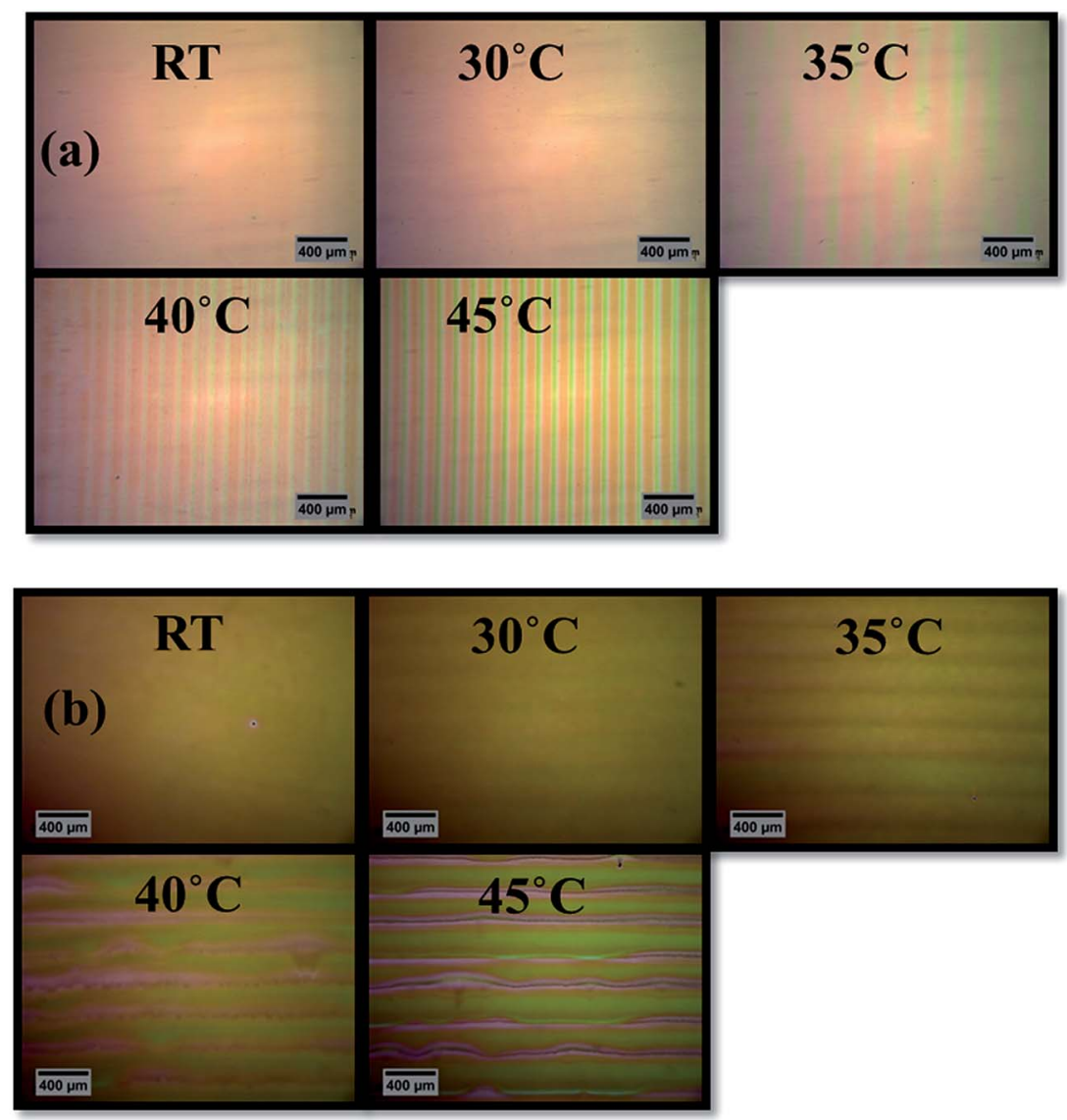

Fig. 4 Optical micrograph of printed PEDOT:PSS on ITO coated glass with two different surface treatment at different substrates temperature (a) oxygen plasma (b) UV-ozone treatment of ITO/glass substrates. 
diameter at higher substrates temperatures. This decrease in the drop diameter restricts the coalescence of different drops at higher substrate temperature and hence results in nonuniformity in the film morphology at higher temperatures. These observations justify the selection of substrate temperature as RT for all further experiments and corroborated the observations made in the previous paragraph.

The profile of the printed drop on the substrate surface is very sensitive to the substrate temperature. Zhou et al. classified different types of shapes depending on substrate temperature. ${ }^{25}$ Lower temperatures typically result in a Gaussian shape whilst high temperatures result in a ring like shape and intermediate temperature lead to a transition shape. ${ }^{\mathbf{1 0}}$ The droplet diameter $\left(D_{\mathrm{m}}\right)$ and drop's maximum height $\left(H_{\mathrm{m}}\right)$ can be calculated using contact angle $(\theta)$ values as shown by following expression ${ }^{26}$

$$
D_{\mathrm{m}}=d_{0} \sin \theta\left\{\frac{1}{\left(3-2 \sin ^{2} \theta / 2\right) \sin ^{4} \theta / 2}\right\}^{1 / 3}
$$

and

$$
H_{\mathrm{m}}=d_{0} \sin ^{2}(\theta / 2)\left\{\frac{1}{(3-2 \sin (\theta / 2)) \sin ^{4} \theta / 2}\right\}^{1 / 3}
$$

where $d_{0}$ is the drop diameter before impact. Based on our contact angle measurements, we have plotted the values of $D_{\mathrm{m}}$ and $H_{\mathrm{m}}$ for various substrate temperatures as shown in Fig. 6 . Here, we observe that with increase in the substrate temperature, (in both case experimental as well as calculated) the droplet diameter decreases even though the experimental drop diameters are different from the calculated values but the observed trend is similar while droplet's central height increases. The droplet was printed on ITO coated glass substrates and due to experimental constraints; it was not possible to measure droplet height using optical profilometer. This is justified by the contact angle measurements, as shown in Fig. 5, which show that the ink spreads for longer time at lower temperature. Our results suggest that the droplet shape is Gaussian in our case.

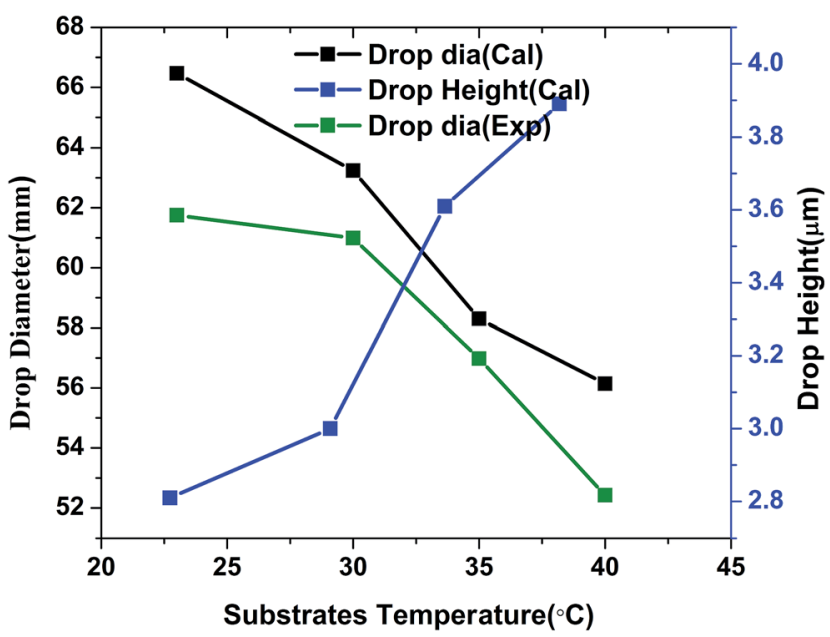

Fig. 6 Relation between the substrate temperature and the final drop diameter (left $Y$-axis) and central height of the droplet (right $Y$-axis).
Due to an increase in the evaporation rate of the solvent from the ink with increase in the substrate temperature, the printed PEDOT:PSS drops dry quickly and hence average drop diameter of the ink also decreases and thus drop to drop interaction decreased, as also reported in the literature. ${ }^{27,28}$ This is quite important as this can lead to premature solidification of the jetted drops by coalescence of the neighboring drops before the film forms. During printing, a fast drying rate of lines reduces the line-to-line interaction. ${ }^{7}$ At very high substrate temperatures, there could be occurrences of internal flow of liquid and hence, the solute redistributes, moving to the periphery leading to the formation of ring like shape from a Gaussian shape. ${ }^{\mathbf{1 0}}$ Similar observations were also made by Subramanian et al. ${ }^{29}$ who showed that with increase in the substrate temperature, the printed line profile changes from convex to concave, affecting the overall printability.

\subsection{Effect of annealing temperature and morphological evolution}

To study the effect of annealing temperature on the morphology of the films, we printed PEDOT:PSS films at different drop spacings from $28 \mu \mathrm{m}$ to $33 \mu \mathrm{m}$ and on substrate pre-treated with oxygen plasma for 3 minutes. The films were subsequently annealed at different temperatures, $100{ }^{\circ} \mathrm{C}$ and $120{ }^{\circ} \mathrm{C}$ for 20 minutes on the hot plate in air. AFM image analysis of the samples with results shown in Fig. 7, showed minimum roughness at $120^{\circ} \mathrm{C}$ for a drop spacing of $30 \mu \mathrm{m}$. Subsequently, we investigated the combined effect of annealing temperature and the drop spacing for all the thicknesses. Overall, we observed that a minimum roughness of PEDOT:PSS films (ca. 4$4.5 \mathrm{~nm}$ ) was achieved at a drop spacing of 30 and $32 \mu \mathrm{m}$ and an annealing temperature of $120^{\circ} \mathrm{C}$ yielding films with a thickness of $c a .120 \mathrm{~nm}$.

A comparison of the morphology with spin coated PEDOT:PSS films of thickness $c a .50 \mathrm{~nm}$, made from the same ink used for printing, shows a porous yet uniform microstructure of the printed films whilst spin coated films show a relatively denser microstructure (see Fig. 8). However, in both cases,

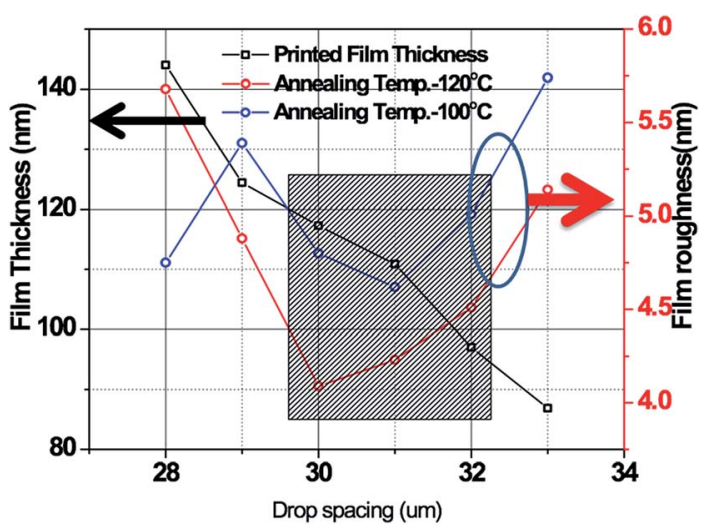

Fig. 7 The figure shows film thickness and film roughness with drop spacing of printed PEDOT:PSS film. Shaded area depicts the parameters optimized for ink-jet printing of PEDOT:PSS ink on oxygen plasma treated ITO/Glass substrates. 
the morphology was uniform across the films with little variations. It is possible that use of centrifugal force in spin coating leads to thinner films with a dense microstructure in the films. As we see subsequently, this difference in the microstructure does seem to adversely affect the device performance to some extent in case of printed films.

\subsection{Integration of printed PEDOT:PSS film into organic solar cell device}

First, we measured the transmission characteristics of printed PEDOT:PSS films which are shown in Fig. 9. Xiong et al. ${ }^{11}$ showed that the addition of additive increases the transmittance after $300 \mathrm{~nm}$ wavelength. The additive facilitates the formation of large PEDOT rich particles and hence, the number of interface between PEDOT:PSS rich particles and PSS lamellas is reduced, which contribute to the reduction in absorbance of light. ${ }^{10,30}$

The figure suggests a maximum transmission of $\sim 89 \%$ at a wavelength of $525 \mathrm{~nm}$ for a PEDOT:PSS film of thickness $\sim 120 \mathrm{~nm}$, which is comparable to that of ITO/glass substrates. The electrical conductivity of printed films was measured by printing PEDOT:PSS ink directly on bare glass substrates to eliminate the effect of ITO because of its high conductivity. The measured values of conductivity were $\sim 0.02 \mathrm{~S} \mathrm{~cm}^{-1}$.

To assess and compare the feasibility of these inkjet printed films on ITO coated glass substrates performance in solar cell devices, we fabricated bulk heterojunction organic solar cells on spin coated as well as inkjet printed PEDOT:PSS having normal device configuration ITO/PEDOT:PSS/P3HT:PC ${ }_{60} \mathrm{BM} / \mathrm{Al}$ with device areas 0.09 and $1 \mathrm{~cm}^{2}$. The current density-voltage $(J-V)$ characteristics were obtained both in dark and light conditions and the results are shown in Fig. 10(a) and (b) and Table 1. The light data on printed films shows poor reverse saturation characteristics which are vital to the shunt resistance of the device. Also, as the dark $J-V$ data shows, the rectification ratio of OSC devices on printed PEDOT:PSS films show smaller rectification ratio $\left(I_{\mathrm{ON}} / I_{\mathrm{OFF}}\right)$ as compared to those on spin coated

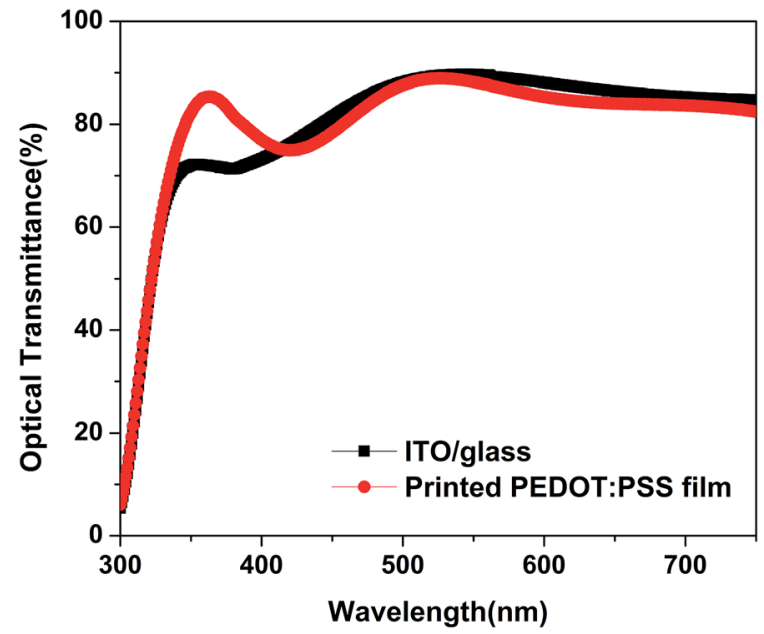

Fig. 9 Optical transmittance measurement of inkjet printed PEDOT:PSS film on ITO/glass substrate. For comparison, spectra of ITO/Glass substrate is also shown.

films. This is indicative of leakage related losses in the devices, mostly attributed to interfacial losses. The results showed that the power conversion efficiency of the device fabricated on inkjet printed PEDOT:PSS was ca. $2.64 \%$ for $0.09 \mathrm{~cm}^{2}$ devices whilst the control device of the same area fabricated on spincoated PEDOT:PSS thin films showed an efficiency of $c a$. $3.10 \%$. As the data in Table 1 suggests, the major contributor to the lower efficiency of the device on printed PEDOT:PSS film is its rather lower fill factor and a smaller $V_{\text {oc }}$. The poor fill factor is attributed to slightly higher sheet resistance and mostly to low shunt resistance of the devices. Latter is attributed to higher thickness of printed PEDOT:PSS films (ca. 120 $\mathrm{nm}$ ) than spin coated PEDOT:PSS films ( $c a .35 \mathrm{~nm}$ ) which results higher roughness of the inkjet printed PEDOT:PSS printed, as evident from the microstructure shown in Fig. 8. This is also the reason why dark current in the OFF state is higher for devices on printed films. Interestingly the $J_{\mathrm{sc}}$ values

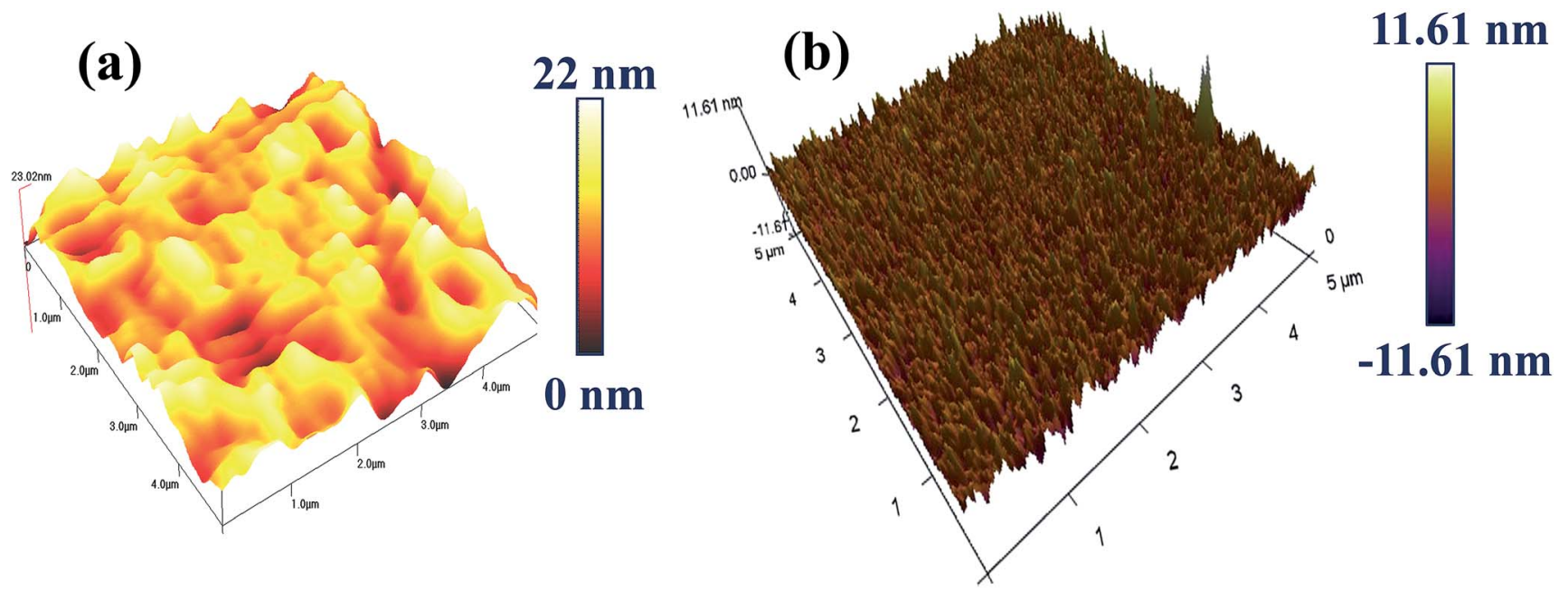

Fig. 8 AFM images of PEDOT:PSS films fabricated using (a) inkjet printing film at drop spacing of $30 \mu \mathrm{m}$ and (b) spin coating. Both the films were fabricated from the same ink. 

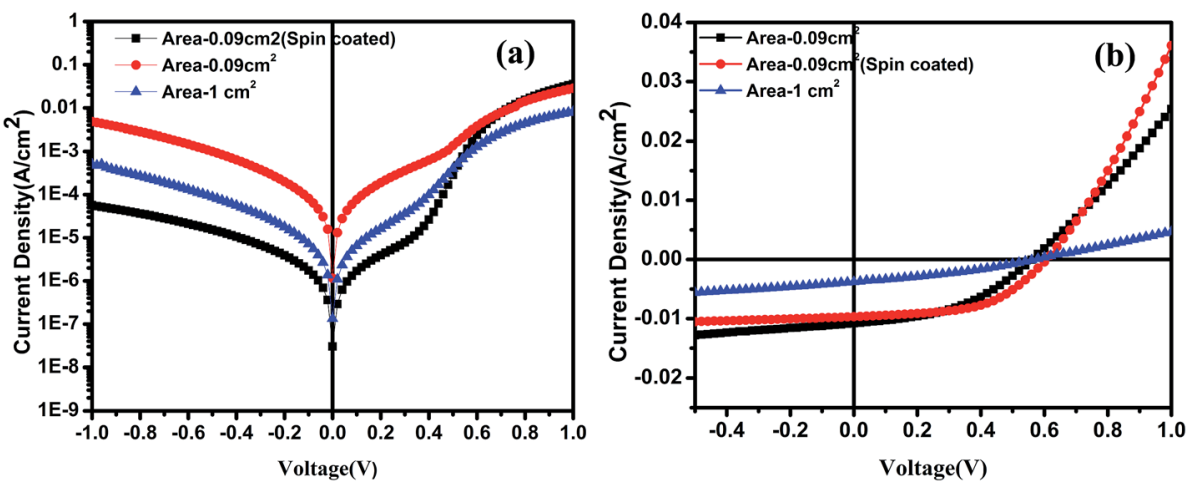

Fig. 10 Current density vs. voltage ( $\mathrm{J}-\mathrm{V}$ ) characteristics of OSC devices fabricated on printed as well as spin coated PEDOT:PSS films: (a) in dark and (b) in light at 1 Sun (1.5 AM).

Table 1 Summary of organic solar cell device characteristics of devices fabricated on inkjet printed and spin coated PEDOT:PSS films

\begin{tabular}{|c|c|c|c|c|c|c|c|}
\hline PEDOT:PSS coating process & $V_{\text {oc }}$ (volt) & $J_{\mathrm{sc}}\left(\mathrm{mA} \mathrm{cm}{ }^{-2}\right)$ & $\mathrm{FF}(\%)$ & PCE (\%) & $R_{\mathrm{S}}(\Omega \mathrm{cm})$ & $R_{\mathrm{sh}}(\Omega \mathrm{cm})$ & Pixel area $\left(\mathrm{cm}^{2}\right)$ \\
\hline Spin coated & 0.6 & $9.63 \pm 0.05$ & $52.3 \pm 0.84$ & $3.02 \pm 0.64$ & 17.46 & 467.28 & 0.09 \\
\hline Inkjet printed & 0.56 & $10.8 \pm 0.35$ & $41.6 \pm 2.66$ & $2.53 \pm 0.11$ & 21.57 & 221.72 & 0.09 \\
\hline
\end{tabular}

of devices on ink-jet printed PEDOT:PSS films was a slightly higher than that in spin coated PEDOT:PSS films. As our results suggested, the printed PEDOT:PSS layer has higher surface roughness as compared to spin coated layer. It is possible that due to higher roughness of inkjet printed PEDOT:PSS layer as well as higher porosity, the amount of P3HT:PCBM deposited is higher than that on spin coated PEDOT:PSS films. This is likely to result in slightly higher current density due to increase light absorption. Further, our experiments on large area devices with pixel size of $1 \times 1 \mathrm{~cm}^{2}$, as shown in Fig. 10(b), suggest that the device performance decreases sharply with increase in the device area to $\sim 0.7 \%$. This is attributed to higher series resistance of the large area device as compared to small area device. It is ${ }^{31}$ shown that with increase in the device area, the sheet resistance increases and hence the power losses increase resulting in inferior performance of large area devices.

Overall, whilst one can see that ink-jet printing indeed can successfully fabricate PEDOT:PSS thin films for OSC devices, there are challenges that remain, particularly to achieve relatively thinner films, down to $\sim 50 \mathrm{~nm}$, with acceptable morphology and desired electrical properties so that device performance is comparable to the devices fabricated on spin coated PEDOT:PSS films. Moreover, one needs to understand the issues related to scaling up to be able to print better devices with larger pixel size, important in realizing full potential of printing.

\section{Conclusions}

In conclusion, we have successfully fabricated $\mathrm{P} 3 \mathrm{HT}: \mathrm{PC}_{60} \mathrm{BM}$ bulk heterojunction organic solar cell devices on ITO coated glass substrates using inkjet printed PEDOT:PSS hole transport layer. To achieve device quality films, we have investigated the effect of the various printing parameters on the printing characteristics of PEDOT:PSS films. Our results suggest that viscosity of the ink strongly affects the drop formation with a viscosity of $5.65 \mathrm{cP}$ leading to the formation of spherical drops without any satellite formation, determined by the ratio of PEDOT:PSS to DI water. We also found film characteristics to be strongly affected by the substrate temperature during printing and annealing temperature as well as surface modification, determined by a mix of surface wetting characteristics, rate of evaporation of the solvent and coalescence of the printed lines. The films showing minimum roughness of $\sim 4$ $\mathrm{nm}$ were printed at room temperature at a drop spacing of 30 $\mu \mathrm{m}$ followed by annealing at $120{ }^{\circ} \mathrm{C}$ leading to a thickness of $c a .120 \mathrm{~nm}$. These films showed a maximum transmittance of $\sim 89 \%$ at wavelength $525 \mathrm{~nm}$ and an electrical conductivity of $2 \times 10^{-2} \mathrm{~S} \mathrm{~cm}^{-1}$. The P3HT: $\mathrm{PC}_{60} \mathrm{BM}$ organic solar cell devices fabricated on the inkjet printed PEDOT:PSS showed a power conversion efficiency of $c a .2 .64 \%$ as compared to $c a$. $3.10 \%$ shown by the devices fabricated on spin coated PEDOT:PSS films using a pixel area of $0.09 \mathrm{~cm}^{2}$. Scaling up of pixel size to 1 $\times 1 \mathrm{~cm}^{2}$ leads to sharp reduction in the device efficiency to $0.7 \%$.

\section{Acknowledgements}

Authors thank Department of Science and Technology, India for the financial support through Indo-UK APEX Project (Grant no. SR/RC-UK/Solar (F)/2010) and DST Nano science and Nanotechnology centers at IIT Kanpur for use of their facilities. 


\section{References}

1 L. Huang, Z. Hu, K. Zhang, P. Chen and Y. Zhu, Thin Solid Films, 2015, 578, 161-166.

2 F. C. Krebs, Sol. Energy Mater. Sol. Cells, 2009, 93, 394-412.

3 D. Jang, D. Kim and J. Moon, Langmuir, 2009, 25, 2629-2635.

4 B. Jo, A. Lee, K. Ahn and S. Lee, Korean J. Chem. Eng., 2009, 26, 339-348.

5 F. Ely, C. O. Avellaneda, P. Paredez, V. C. Nogueira, T. E. A. Santos, V. P. Mammana, C. Molina, J. Brug, G. Gibson and L. Zhao, Synth. Met., 2011, 161, 2129-2134.

6 P. Wilson, C. Lekakou and J. F. Watts, Org. Electron., 2012, 13, 409-418.

7 S. H. Eom, S. Senthilarasu, P. Uthirakumar, S. C. Yoon, J. Lim, C. Lee, H. S. Lim, J. Lee and S.-H. Lee, Org. Electron., 2009, 10, 536-542.

8 W. Wang, M. A. Ruderer, E. Metwalli, S. Guo, E. M. Herzig, J. Perlich and P. Müller-Buschbaum, ACS Appl. Mater. Interfaces, 2015, 7, 8789-8797.

9 J. Ouyang, Q. Xu, C.-W. Chu, Y. Yang, G. Li and J. Shinar, Polymer, 2004, 45, 8443-8450.

10 Y. Xia, K. Sun, J. Chang and J. Ouyang, J. Mater. Chem. A, 2015, 3, 15897-15904.

11 Z. Xiong and C. Liu, Org. Electron., 2012, 13, 1532-1540.

12 H. Song, C. Liu, J. Xu, Q. Jiang and H. Shi, RSC Adv., 2013, 3, 22065-22071.

13 Y. Liu, Z. Song, Q. Zhang, Z. Zhou, Y. Tang, L. Wang, J. Zhu, W. Luo and W. Jiang, RSC Adv., 2015, 5, 45106-45112.

14 S. Liu, H. Deng, Y. Zhao, S. Ren and Q. Fu, RSC Adv., 2015, 5, 1910-1917.

15 M. S. A. Sher Shah, S. Muhammad, J. H. Park, W.-S. Yoon and P. J. Yoo, RSC Adv., 2015, 5, 13964-13971.

16 H. Wang and V. Kumar, RSC Adv., 2015, 5, 9650-9657.
17 M. W. Rowell and M. D. McGehee, Energy Environ. Sci., 2011, 4, 131-134.

18 H. Jin, A. Pivrikas, K. H. Lee, M. Aljada, M. Hambsch, P. L. Burn and P. Meredith, Adv. Energy Mater., 2012, 2, 1338-1342.

19 J. D. Servaites, M. A. Ratner and T. J. Marks, Energy Environ. Sci., 2011, 4, 4410-4422.

20 A. Armin, I. Kassal, P. E. Shaw, M. Hambsch, M. Stolterfoht, D. M. Lyons, J. Li, Z. Shi, P. L. Burn and P. Meredith, J. Am. Chem. Soc., 2014, 136, 11465-11472.

21 A. J. Moulé, J. B. Bonekamp and K. Meerholz, J. Appl. Phys., 2006, 100, 094503.

22 H. Jin, C. Tao, M. Velusamy, M. Aljada, Y. Zhang, M. Hambsch, P. L. Burn and P. Meredith, Adv. Mater., 2012, 24, 2572-2577.

23 J. E. Fromm, IBM J. Res. Dev., 1984, 28, 322-333.

24 J. S. Kim, R. H. Friend and F. Cacialli, J. Appl. Phys., 1999, 86, 2774-2778.

25 J. Zhou, J. H. Fuh, H. Loh, Y. Wong, Y. Ng, J. Gray and S. Chua, Int. J. Adv. Des. Manuf. Technol., 2010, 48, 243-250.

26 M. Ikegawa and H. Azuma, JSME Int. J., Ser. B, 2004, 47, 490496.

27 A. Teichler, R. Eckardt, C. Friebe, J. Perelaer and U. S. Schubert, Thin Solid Films, 2011, 519, 3695-3702.

28 A. M. J. van den Berg, A. W. M. de Laat, P. J. Smith, J. Perelaer and U. S. Schubert, J. Mater. Chem., 2007, 17, 677-683.

29 D. Soltman and V. Subramanian, Langmuir, 2008, 24, 22242231.

30 I. Cruz-Cruz, M. Reyes-Reyes, M. A. Aguilar-Frutis, A. G. Rodriguez and R. López-Sandoval, Synth. Met., 2010, 160, 1501-1506.

31 S. Choi, W. J. Potscavage and B. Kippelen, J. Appl. Phys., 2009, 106, 054507. 